\author{
Muriel Boucart · Irving Biederman · Christine Cuervo • \\ Jean-Marie Danion · Johan Wagemans
}

\title{
Effect of benzodiazepines on structural and conceptual/lexical priming
}

Received: 23 January 2002 / Accepted: 8 August 2002 / Published online: 25 October 2002

(C) Springer-Verlag 2002

\begin{abstract}
Rationale: Impaired perceptual priming, as assessed by naming reaction times and accuracy, for briefly presented contour-deleted pictures under lorazepam has been documented in several studies but whether the nature of this impairment is visual versus conceptual/ lexical is not clear. We used a previously developed paradigm to examine whether lorazepam affects visual processes involved in the coding of contour information or conceptual/lexical representations. Method: Three groups were tested (lorazepam, diazepam and placebo). In the study phase, participants named line drawings, presented for $500 \mathrm{~ms}$, in which $50 \%$ of the contour was deleted by removing every other line and vertex from each part. In the test phase, participants saw the identical picture, its complement (the other $50 \%$ of the contour), or a samename/different-shape picture, each presented for $200 \mathrm{~ms}$. Results: For all three groups, the magnitude of priming, as assessed by naming RTs and error rates, was equivalent in the identical and in the complementary conditions and the amount of facilitation was reduced in the same-name condition. Perceptual priming occurred both in the lorazepam and in the diazepam groups, though reduced, compared to placebo, for RTs. No conceptual priming (i.e. facilitated performance in the same-name condition) was observed in the benzodiazepine groups. Conclusion: Equivalent priming for identical and complementary pictures in the three groups suggests that
\end{abstract}

\section{Boucart (}

CHRU Lille, Hopital Roger Salengro,

Exploration Fonctionnelle de la vision, 59037 Lille, France

e-mail: m-boucart@chru-lille.fr

\section{Biederman}

Department of Psychology, University of Southern California, Hedco Neuroscience Building, MC2520, Los Angeles,

CA 90089-2520, USA

C. Cuervo - J.-M. Danion

Dept Psychiatrie I, Hopitaux Universitaires de Strasbourg,

INSERM 405, 67091 Strasbourg Cedex, France

\section{J. Wagemans}

Department of Experimental Psychology, University of Leuven, Tiensestraat 102, 3000 Leuven, Belgium benzodiazepines do not affect the activation of the geon structural description. The lack of priming for same-name pictures suggests that benzodiazepines affect access, retrieval or selection of conceptual/lexical information.

Keywords Benzodiazepines - Diazepam - Lorazepam • Object recognition $\cdot$ Perceptual priming $\cdot$ Conceptual priming

\section{Introduction}

A number of studies have documented a deficit of priming under lorazepam, a benzodiazepine (Danion et al. 1992; Curran and Gorenstein 1993; Schifano and Curran 1994; Stewart et al. 1996; Buffet-Jerrott et al. 1998). Priming is the facilitation of processing a stimulus as a function of its prior exposure. For example, a task often used in implicit memory priming studies is word-stem completion, in which participants are presented with three letter word beginnings (e.g. BLA) and asked to complete them with the first word that comes to mind. Implicit memory is indicated when participants complete a stem more often with a word they have previously studied on a presented list (e.g. BLANK) than with a non-presented word (BLAME). Word-stem completion is impaired by lorazepam, both when the stimuli are presented visually (Danion et al. 1989; Curran and Gorenstein 1993) or by ear (Vidailhet et al. 1999). Impaired priming under lorazepam has later been generalized to another task considered to tap priming: picture completion (Sellal et al. 1992; Vidailhet et al. 1994; Legrand et al. 1995). In these studies, participants are presented with a series of line drawings of objects in a study phase for $10 \mathrm{~s}$ each that they are asked to name. In a test phase occurring several minutes later, they are presented with a series of increasingly more complete line drawings for $5 \mathrm{~s}$ each, including pictures presented at study (old pictures) and pictures that were not presented at study (new pictures). Again, priming was found for fragmented pictures corresponding to the complete version seen in the study 
phase for placebo-treated participants but not for lorazepam-treated participants.

A lack of priming for lorazepam-treated participants on picture-completion tasks could have resulted from impairment at a number of stages in the processing of a stimulus. One possibility is that lorazepam interferes with early perceptual processing, such as that required for the grouping or the storage of the image elements in the incomplete forms (Giersch et al 1995, 1996; Giersch 1999; Elliott et al. 2000; Beckers et al. 2001). Another possibility is that the reduction in priming is not a consequence of impairment of early perceptual processes for grouping, but on the later processes of conceptual or lexical access, so that the storage or retrieval of the object's concept or its name is impaired. Still a third possibility is that the impairment is at an intermediate level where, for example, a structural description of the object's parts and relations is specified (Biederman 1987; Hummel and Biederman 1992).

Previous studies have compared priming for identical (old) pictures and pictures never seen in the study phase (new pictures) (Vidailhet et al. 1994; Legrand et al. 1995). Although the comparison of old versus new pictures isolates the effect of the lorazepam as a priming effect (rather than just a benefit of general practice of the task), the stage or stages that are impaired remain uncertain. That is, one cannot isolate the benefits of repetition to early processing by which local image features are grouped or stored, an intermediate representation (a structural description), or storage or retrieval of the object's basic level concept or name, i.e. conceptual or lexical access. The current experiment was designed to assess the locus of the effect of the benzodiazepines on priming. We used a paradigm devised by Biederman and Cooper (1991) to examine whether the benzodiazepines (lorazepam and diazepam) affect: a) the early coding of local image features, e.g. edges and vertices, b) the activation of an intermediate representation of the object, such as its structural description, or c) storage or access to conceptual/lexical representations.

\section{Geon structural descriptions}

Biederman (1987) proposed a model of how objects can be recognized in a fraction of a second despite variations in size, orientation in depth and spatial position. According to this theory, the edges corresponding to the orientation and depth discontinuities of an object are characterized according to qualitative differences in their viewpoint invariant characteristics, such as straight versus curved, parallel versus non-parallel, and the type of vertices formed by the co-termination of edges. The edges activate units representing simple shape primitives, called "geons", such as cones, blocks, wedges, cylinders, circles, and rectangles, that can be distinguished from each other independent of orientation in depth. Objects are represented by a structural description, termed a geon structural description (GSD), that specifies the pairwise relations (e.g. top-of, larger-than, etc.) between the geons and attributes of the geons such as orientation (e.g. vertical) and qualitative aspect ratio (e.g. whether the diameter of the cross section is smaller than, larger than or about the same size as the axis of the geon). According to the theory, the geons are activated by local image features such as vertices and edges, but the memory representation is of the GSD. By representing the object in terms of the simple parts rather than local image features or an overall template of the object, near invariance can be achieved at different orientations of the object or different occlusion patterns, as long as the same parts and relations can be extracted from the image.

To test experimentally the issue of local features versus parts, Biederman and Cooper (1991) created pairs of complementary versions of object pictures by deleting every other edge and vertex from each part (or geon) to create two images of each object (see Fig. 1). The two images for each object, when superimposed, would form an intact picture with no overlap in contour. The complementary images were created in such a way that each part (or geon) of the object could be recovered (or fail to be recovered) from each of the images. Although complementary images shared no edges and vertices, they presumably would activate the same geons. Because the amount of contour deleted from each image was substantial and included vertices, it is unlikely that a local process of good continuation could have completed the contour of these images. If identical and complementary images were to prime each other equally, it would be evidence against the locus of priming being at a stage that specified local image elements.

In the study phase, Biederman and Cooper's (1991) participants named, as quickly as possible, a number of brief $(500 \mathrm{~ms})$ presentations of one member from each complementary pair. On the second block (the test phase), they saw the identical image, its complement, or a samename different/shape exemplar (also contour-deleted) from a category with the same-name and basic level concept but with a different shape, such as that shown in the rightmost column of Fig. 1. The images were shown for $200 \mathrm{~ms}$ on the second block. Naming RTs and error rates were markedly lower to the identical exemplars than to the same-name/different-shape exemplars, indicating that a portion of the priming was visual and not just conceptual or lexical. The critical comparison, however, concerned the complementary condition. If priming was a function of repetition of the specific vertices and edges in the image, then the complementary condition would have been equivalent to the same-name/different-exemplar condition, as neither shared any local features with the original image. Remarkably, there was no difference in performance in naming complementary and identical images, indicating that none of the priming could be attributed to the specific vertices and lines actually present in the image. Insofar as the stimuli in the different exemplar condition had the same basic-level category (and name) as the stimuli in the identical and complementary conditions, the advantage of the identical and 


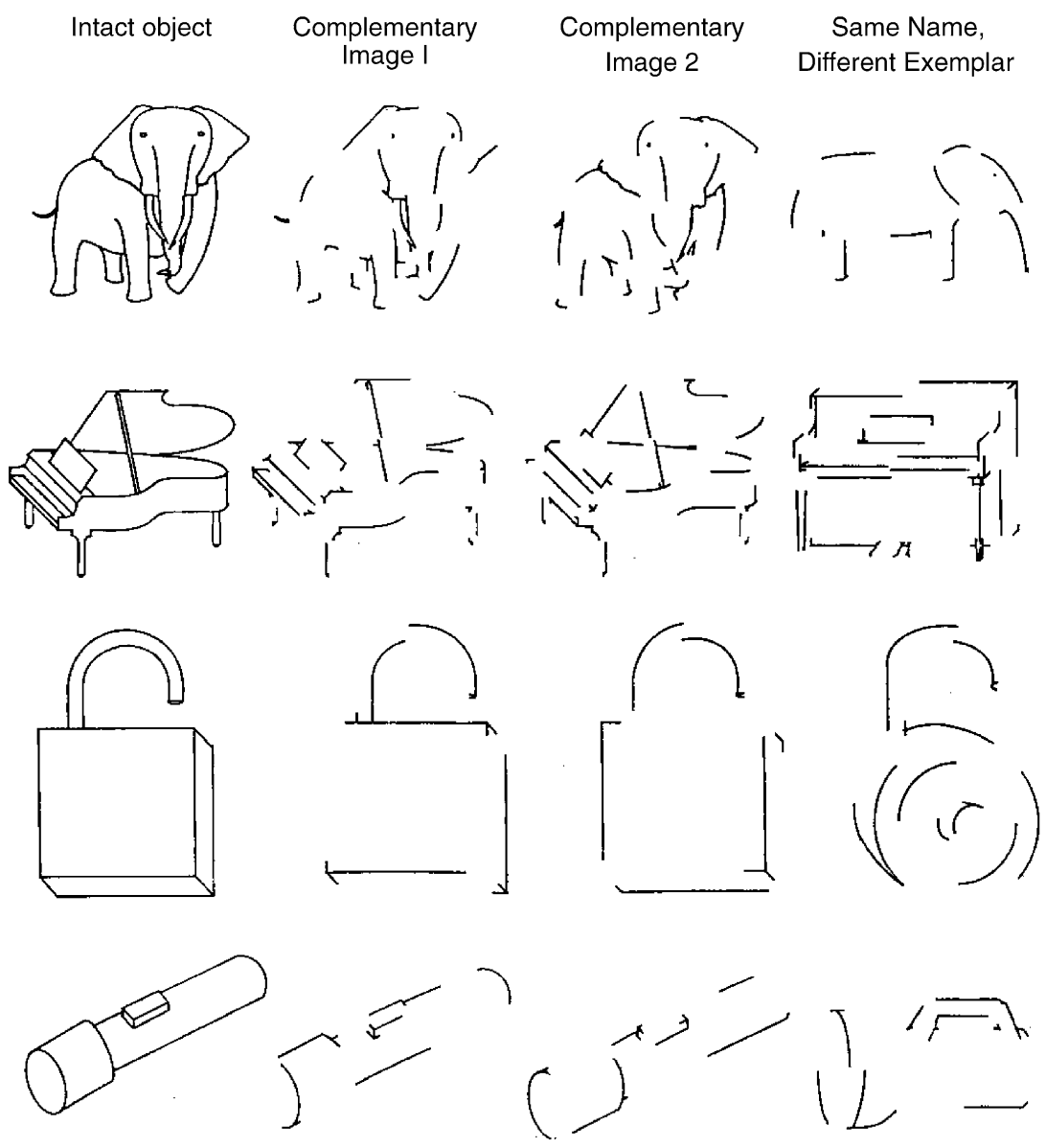

Fig. 1 Examples of four of the experimental stimuli. Second and third columns: a complementary pair of contour-deleted images for the object in the first column. These images were produced by deleting every other vertex and edge from each object part. (Very long lines, as those in the upright piano, were split into two lines.) If the members of a complementary pair were superimposed they would produce the intact image shown in the first column (which the participants never saw) without any overlap in the pixels.

complementary conditions could not be attributed to activation of a basic level concept or name.

Before concluding that the priming was mediated by an intermediate representation specifying the object's parts and relations, which differed between the stimuli in the identical and complementary conditions compared to the different exemplar condition, it is necessary to rule out the possibility of subordinate level concept priming. If priming was due to subordinate level concept activation of, for example, a grand piano rather than just piano, then the advantage of the identical and complementary conditions could have been obtained without a contribution from the structural description. To test this possibility, Biederman and Cooper designed a second experiment in which complementary images were created by deleting half the parts of the objects. With these stimuli, presumably, the same subordinate category would be activated from either member of a complementary pair, but through different parts. As with the first experiment, performance
Fourth column: an example of a same-name/different-shaped exemplar which served as a control for lexical and basic level concept priming for participants who viewed one of the two complementary images on their first block. There was a complementary member for this exemplar as well (not shown) which allowed the exemplar to serve as a priming stimulus for half the participants so that the images that were used to assess priming and name-concept controls were completely balanced over participants

with the identical images was better than with the different exemplars, indicating that none of the priming could be attributed to a subordinate conceptual model. Critically important was that the complementary and different exemplar conditions were equivalent, indicating that none of the priming in the first experiment could be attributed to access to a subordinate level concept. The results of the two experiments, taken together, suggest that all of the priming can be attributed to an intermediate representation specifying the parts (and their relations) of the object; none to the activation of the local image features or subordinate or basic level concepts or names.

Present experiment: the effects of benzodiazepines on object priming

Biederman and Cooper's (1991) paradigm was used to provide a better understanding of the mechanisms of the 
deleterious effect of the benzodiazepines on perceptual priming. Relative to the placebo control, (a) diminished priming for identical fragmented pictures, relative to same-name, different-shaped pictures, would indicate that the benzodiazepines reduce perceptual priming; (b) priming in the identical condition but not in the complementary condition would be evidence that the benzodiazepines interfere with the activation of the geon structural description; and (c) a priming effect for identical and complementary conditions and a lack of priming for same-name/different-exemplar pictures would indicate that the benzodiazepines impair conceptual/lexical priming.

In addition to the three priming conditions, we compared performance for two benzodiazepines (lorazepam and diazepam). Previous studies (Sellal et al. 1992; Vidailhet et al. 1994) have shown that a dose of $2.5 \mathrm{mg}$ lorazepam interfered with performance in both an explicit memory task (free recall) and implicit memory (perceptual priming) but that diazepam at an equivalently sedative dose did not interfere with implicit memory. A possible issue of concern in studies using drugs is that of sedation. The diazepam group was introduced to provide a control for non-specific factors such as sedation. If both drugs induce similar sedative effects when only one drug exhibits a deleterious effect on priming, then this deleterious effect could not be attributed to non-specific factors. From the previous results with diazepam and lorazepam, we might expect reduced priming only with lorazepam, not diazepam. In particular, given the effects reported by Giersch et al. $(1995,1996)$, we would expect to see the effects of lorazepam, relative to diazepam, primarily confined to a reduction in the priming of intermediate representations. That is, the complementary and the different exemplar conditions would be equivalent and both would be inferior to the identical condition.

\section{Materials and methods}

\section{Participants}

The study was approved by the Ethical Committee of Alsace. Sixtysix healthy volunteers (18-35 years of age) were recruited from the Faculty of Medicine of the University Louis Pasteur in Strasbourg. They were native French speakers. They had no medical illness or history of alcoholism, drug abuse or tobacco consumption of more than ten cigarettes/day. They were not chronic users of benzodiazepines, nor had they taken any concomitant medication for at least 21 days. Participants were instructed to abstain from beverages containing caffeine or alcohol for the $24 \mathrm{~h}$ prior to the study. All participants were tested in the morning after an overnight fast. They all had normal or corrected-to-normal vision. Written informed consent was obtained from all volunteers before they entered the study. Participants were paid 1000 Francs for their participation.

Stimuli

The pictures were 24 pairs of common objects, each with a readily available basic level name, drawn in Cricket Draw, from Bieder- man and Cooper (1991). Members of a pair had the same basic level name but different part compositions, such as a grand piano and an upright piano. An effort was made to have the two exemplars of a class appear as dissimilar as possible, subject to the constraint that both versions be readily identifiable. Two complementary versions of each of the 48 pictures were prepared by deleting every other edge and vertex from each part of the 48 object pictures as shown in Fig. 1. When edges were deleted, a small portion of the edge was retained to define the adjacent vertex. Because deleting the long sides of a large component seriously impaired the ability to identify that object at brief durations, long edges (equal or greater than 1.65 degrees) were considered as two separate edges. Each version contained approximately $50 \%$ of the contour from each component. Each image thus contained half the contour from the original intact object. The two versions, when superimposed, formed an intact picture without any overlap in contour. The contour deletion procedure typically allowed the same components to be identified in both members of a pair, such as the block that forms the body of the lock in Fig. 1. The detailed set of rules for creating the images are described in Biederman and Cooper (1991).

\section{Apparatus}

The stimuli were centrally displayed on the color screen (14 inch) of a 486 PC computer equipped with a VGA graphic card. Viewing distance was fixed at $57 \mathrm{~cm}$. The screen resolution was $640 \times 480$ pixels for 256 colors. The maximum extent of each picture could be contained in a square whose sides subtended a visual angle of 5.6 degrees. The refresh rate of the screen was $16.7 \mathrm{~ms}$. Participants responded by speaking into a microphone, which triggered a voice key connected to the computer.

\section{Experimental procedure and drugs}

Participants were randomly assigned to one of three parallel groups of 22 participants each: a placebo (lactose $190 \mathrm{mg}$ ) group, a lorazepam $0.038 \mathrm{mg} / \mathrm{kg}$ group and a diazepam $0.3 \mathrm{mg} / \mathrm{kg}$ group. The diazepam and lorazepam doses were equally sedative (Dundee et al. 1979). Drugs were administered orally using a double-blind procedure. In order to evaluate the effects of each drug at its peak plasma concentration, which is usually attained within 60 min after oral administration for diazepam and within $120 \mathrm{~min}$ for lorazepam (Mandelli et al. 1978; Greenblatt 1981), a double-placebo procedure was used. Participants in the diazepam group received placebo at 7.30 a.m. and diazepam at 8.30 a.m.; participants in the lorazepam group received lorazepam at 7.30 a.m. and placebo at 8.30 a.m.; participants in the placebo group received placebo at both times.

The pictures were presented in two blocks of trials, a study phase (block 1) and a test phase (block 2). Approximately 7 min intervened between study and test. On both blocks the participants named with the basic level name (e.g. "piano") each picture as it was shown. To decrease the likelihood of participants using other names for the stimuli, prior to the experiment, participants were presented with the names of the objects. On the first block pictures were displayed centrally for $500 \mathrm{~ms}$ each and on the second block they were shown for $200 \mathrm{~ms}$. These durations were selected to allow reasonably high rates of identification on block 1 of the contour deleted stimuli and only a single fixation on block 2 . Because of the shortened presentation duration on block 2, priming as well as general improvement effects are underestimated. In both blocks each stimulus was followed by a random appearing arrangement of lines that served as a mask. The mask was displayed for $500 \mathrm{~ms}$. The naming RTs were recorded with a voice key. 
Design

In the study phase, participants viewed one member of each of the 24 basic level pairs. On the test phase, for each object viewed on the first block, participants could see the identical image that had been shown on the first block, its complement with the remaining edges and vertices, or a different exemplar with the same name. These three types of block 2 trials were equally distributed over the three conditions (hence, eight objects per condition) with different participants having different objects in the various conditions. The sequences of images were balanced across participants so that the mean serial position of every image in every condition was the same, with all members of a pair of objects and the complements of each member serving equally often as priming and primed stimuli and equally often in the three block 2 conditions.

\section{Results}

The results are shown in Fig. 2. RTs and accuracy for naming pictures in block 1 did not differ significantly among the three groups $[F(2,63)=1.37$, n.s. for RTs and $F(2,63)=0.8$, n.s. for errors], although the three groups were ordered the same on both measures, with the Placebo group showing the best performance (shortest RTs and lowest error rates) and the Diazepam group the worst. With one exception (the same-name/different exemplar condition for RTs), these orderings were maintained over all block 2 conditions for both measures. A speed-accuracy trade-off for the group ordering can thus be ruled out. The overall improvement in performance from block 1 to block 2 was primarily shown in the RTs, not in the error rates. RTs were longer in the study phase (block 1) than in the test phase (block 2) [1262 versus $1158 \mathrm{~ms}, F(1,63)=37.4, P<0.001]$. The reduction in error rates from $18.8 \%$ in the study phase to $17.4 \%$ in the test phase fell short of significance $[F(1,63)=2.98$, $P<0.088]$. The fact that accuracy did not improve significantly from block1 to block 2 can be attributed to the shorter exposure time in block $2(200 \mathrm{~ms})$ as compared to block 1 (500 ms).

Averaged over block 1 and block 2, the main effect of group was significant for RTs but not for errors [placebo: $1098 \mathrm{~ms}$ (15.8\% errors), diazepam: $1237 \mathrm{~ms}(19.4 \%)$, and lorazepam: $1217 \mathrm{~ms}(18.3 \%) ; F(2,63)=4.83, P<0.011$ for RTs and $F(2,63)=2.2$, n.s for errors]. There was also a main effect of condition [block 1: $1262 \mathrm{~ms}(18.8 \%)$, identical: $1126 \mathrm{~ms}$ (16.6\%), complementary: $1125 \mathrm{~ms}$

Table 1 Magnitude of priming of RTs as a function of group and condition. All entries are the difference in RTs between block 1 and the score in the condition indicated in block 2 (block 1 minus block 2 ). The negative priming value means that the RTs on block 2 in that condition (lorazepam, same name) were longer than the block 1 RTs. Same name is a measure of non-visual priming. Visual priming was calculated by subtracting the mean same-name RTs
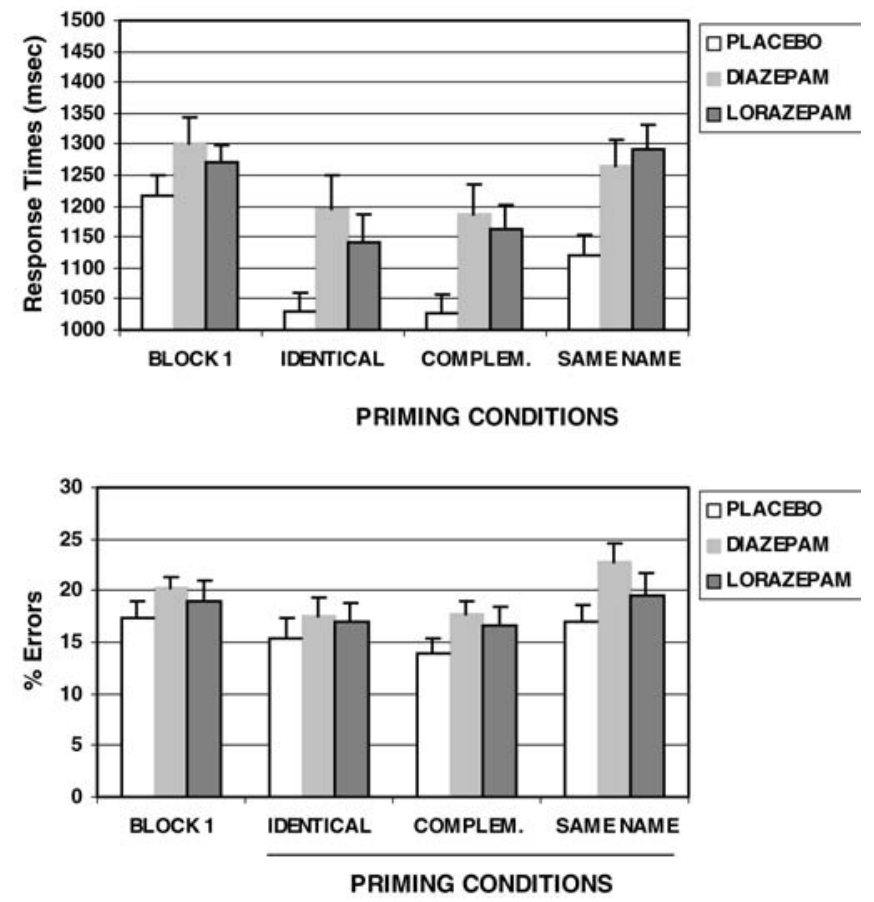

Fig. 2 Mean RTs of correct responses and mean error rates as a function of the block (study versus test phase) and the priming condition (identical, complementary and same-name/different-exemplar) for each treatment group

$(16.1 \%)$, same name: $1225 \mathrm{~ms}(19.8 \%), F(3,189)=26.44$ $P<0.001$ for RTs and $F(3,189)=5.54, P<0.001$ for errors $]$.

\section{Priming}

Priming for the different groups was evaluated by subtracting the block 2 scores from the block 1 scores as shown in Table 1 for RTs and Table 2 for error rates. These scores thus take into account the initial block 1 differences, even though these fell short of significance. As with the block 1 differences, most of the differences arose in the RTs, rather than the error rates. It must be reemphasised that all groups showed substantial facilitation insofar as the second block presentation durations were only $200 \mathrm{~ms}$.

from the mean of the RTs in the identical and complementary conditions. The mean priming for each group was calculated over identical, complementary, and same-name conditions (mean 1-3). Significance levels refer to the difference between RTs in block1 and RTs in each priming condition (identical, complementary and same-name) in block 2

\begin{tabular}{lllrrr}
\hline Group & Identical & Complementary & Same name & Visual priming & Mean $(1-3)$ \\
\hline Placebo & $187 \mathrm{~ms}(P<0.001)$ & $190 \mathrm{~ms}(P<0.001)$ & $98 \mathrm{~ms}(P<0.001)$ & $91 \mathrm{~ms}$ & $158 \mathrm{~ms}$ \\
Diazepam & $104 \mathrm{~ms}(P<0.02)$ & $114 \mathrm{~ms}(P<0.012)$ & $35 \mathrm{~ms}($ n.s. & $74 \mathrm{~ms}$ & $84 \mathrm{~ms}$ \\
Lorazepam & $127 \mathrm{~ms}(P<0.012)$ & $108 \mathrm{~ms}(P<0.010)$ & $-21 \mathrm{~ms}($ n.s $)$ & $139 \mathrm{~ms}$ & $71 \mathrm{~ms}$ \\
Mean & $139 \mathrm{~ms}$ & $137 \mathrm{~ms}$ & $37 \mathrm{~ms}$ & &
\end{tabular}


Table 2 Magnitude of priming of error rates as a function of group and condition. All entries are the difference in error rates between block 1 and the score in the condition indicated in block 2 (block 1 minus block 2). Negative priming values means that the error rates on block 2 were higher than the block 1 error rates. Same name is a measure of non-visual priming. Visual priming was calculated by subtracting the mean same-name error rates from the mean of the

\begin{tabular}{llllll}
\hline Group & Identical & Complementary & Same name & Visual priming & Mean (1-3) \\
\hline Placebo & $2 \%$ (n.s.) & $3.2 \%(P<0.02)$ & $0.4 \%$ (n.s.) & $2.25 \%$ & $1.9 \%$ \\
Diazepam & $2.7 \%$ (n.s.) & $2.5 \%$ (n.s.) & $-2.5 \%$ (n.s.) & $0.1 \%$ & $0.9 \%$ \\
Lorazepam & $2.1 \%$ (n.s.) & $2.3 \%$ (n.s.) & $-0.6 \%$ (n.s.) & $1.6 \%$ & $1.3 \%$ \\
Mean & $2.3 \%$ & $2.7 \%$ & $-0.9 \%$ & & \\
\hline
\end{tabular}

error rates in the identical and complementary conditions. The mean priming for each group was calculated over identical, complementary, and same-name conditions (mean 1-3). Significance levels refer to the difference between error rates in block1 and error rates in each priming condition (identical, complementary

and same-name) in block 2
Consistent with previous results, both drug groups showed reduced priming overall, relative to the placebo group $[F(2,63)=3.73, P<0.029]$. Perceptual priming was calculated by subtracting the mean RTs in the identical and complementary conditions from the same-name/ different-shape condition. Same name is a measure of non-visual (conceptual) priming. For RTs, the diazepam group showed more conceptual priming (by $56 \mathrm{~ms}$ ) and less perceptual priming (by $65 \mathrm{~ms}$ ) than the lorazepam group. Remarkably, the lorazepam group showed greater perceptual priming than even the placebo group, by $48 \mathrm{~ms}$. This pattern of results produced a significant group $\times$ priming conditions (perceptual/conceptual) interaction for RTs $[F(2,63)=3.4, P<0.043$ for RTs, but not for errors $F<1]$. This interaction resulted mainly from the same-name/ different-shape priming condition $[F(2,63)=3.3, P<0.04]$.

All three groups replicated the general pattern of results reported by Biederman and Cooper (1991): Substantial and equivalent priming for the identical and complementary conditions, both of which showed more priming than the same-name condition.

\section{Discussion}

The main results can be summarized as follows: (1) Biederman and Cooper's (1991) results were replicated in all three groups: the magnitude of priming was equivalent in the identical and in the complementary conditions and the amount of facilitation was reduced in the same-name/ different-exemplar condition; (2) perceptual priming occurred both in the lorazepam and the diazepam groups for RTs in that the identical, as well as the complementary, conditions had reduced RTs compared to the samename/different-exemplar condition; (3) the benzodiazepine groups evidenced reduced priming overall but especially for conceptual/lexical priming in that the magnitude of priming for the same-name/different-shape condition was markedly lower than in the placebo group. The reduced conceptual/lexical priming was particularly apparent in the lorazepam group.

The equivalent magnitude of priming for identical and complementary images indicates that all the perceptual priming in object naming can be attributed to activation of the components (geons) in the image. None of the priming here can be attributed to explicit image features, the vertices and edges that were present in the image, since there was no advantage for the identical over the complementary condition, nor to the specific object model given that the magnitude of priming was smaller for the same-name/different-exemplar condition than for the complementary condition. The fact that the same pattern of result occurred both in the placebo group and in the benzodiazepine groups suggests that benzodiazepines did not affect the activation of the geon structural description. The construction of an intermediate representation does not seem to be impaired by benzodiazepines.

We found perceptual priming for both diazepam- and lorazepam-treated participants, though the magnitude was much reduced as compared to the placebo group. This finding, at first glance, contrasts with previous reports that lorazepam interferes with perceptual priming, with the identification of incomplete pictures (Sellal et al. 1992; Vidailhet et al. 1994; Legrand et al. 1995). The results of the present experiment can be readily reconciled with these prior reports. These studies employed a task, identification of fragmented pictures, which, at first glance, might be assumed to tap early perceptual processes. However, the design of these prior studies did not allow the isolation of the particular stage affected by a drug. For instance, in these studies participants were presented with incomplete pictures for a longer exposure time as that used in the present experiment (e.g. 5 or $10 \mathrm{~s} /$ picture), or they were presented with a series of more and more complete picture with an ascending method of limits. These conditions were more susceptible to trigger the identification process and tap lexical priming. Lowered identification performance could have been produced through interference at the early, intermediate, or at late stages mediating identification. If the deleterious effects of lorazepam were on the late (conceptual/lexical) stage of identification in previous studies, then the prior results would be generally compatible with the results of the present experiment. Indeed, we found reduced conceptual/lexical priming for benzodiazepine treated participants. These results are not only consistent with those of prior conceptual/lexical priming tasks (Danion et al. 1989; Curran and Gorenstein 1993; Schifano and Curran 1994; Stewart et al. 1996; Buffet-Jerrott et al. 1998; Buffet- 
Jerrott and Stewart 2002), they are also consistent with the results of Brown et al. (1989), who reported conceptual priming to be suppressed in participants administered with a single high dose of lorazepam.

In contrast to perceptual priming, conceptual priming is supposed to occur when there is no perceptual similarity between the primed stimulus and the test cue. Pharmacological studies investigating conceptual priming classically use a category generation task. Participants are presented with a list of words in a study phase. Conceptual priming is demonstrated by facilitation in performance resulting from presentation of a test cue (e.g. "name an animal") that is conceptually related to a studied item (e.g. elephant). Tulving and Schacter (1990) suggested that conceptual and perceptual priming are subserved by separate memory systems. Conceptual priming is based on the semantic memory system whilst perceptual priming is based on the perceptual representation system. Results from pharmacological studies investigating the effect of benzodiazepines on conceptual priming have revealed some inconsistencies. For instance, Brown et al. (1989) found priming to be suppressed in participants administered with a single dose of lorazepam in a category generation task. This is in contrast with another study by Bishop and Curran (1998), who reported that lorazepam-treated participants performed as well as placebo-treated participants in a category generation task suggesting that conceptual priming is preserved under lorazepam. To explain this difference, the authors suggested that Brown et al.'s participants were provoked into explicit retrieval during the priming task because they were asked to rate each exemplar for the likelihood that it was included in the priming list.

The conceptual priming condition used in the present experiment (same-name exemplars) and the category generation task are difficult to compare. Category generation uses words and assesses knowledge about semantic categories (e.g. "name a type of tree"), whilst our study requires access to basic category representation through perceptual identification of degraded pictures. Evidence from neuropsychological studies indicates that some patients might exhibit a differential pattern of semantic impairment for words and pictures. For instance, Bub et al. (1988) reported the case of a patient MP who has access to knowledge derived from the basic-level concept with pictures as stimuli but whose comprehension of words is severely impaired, though other patients might display impaired visual object recognition alongside relatively intact visual word recognition (Humphreys and Rumiati 1998), suggesting that the structural description subserving object identification may differ from those involved in word identification.

Consistent with our study, Thiel et al. (2001) tested the effect of lorazepam (participants were given $2 \mathrm{mg}$ lorazepam orally) on word-stem completion using event-related fMRI. Placebo-treated participants showed repetition suppression (decreased neuronal response) in the left extrastriate, left middle frontal gyrus and left inferior frontal gyrus associated with improved perfor- mance for old words (behavioral measure of priming). Compared to placebo-treated participants, priming was attenuated though not abolished in lorazepam-treated participants but drug impaired repetition suppression in frontal areas involved in semantic processing. Left inferior frontal cortex is activated by semantic word classification, word generation and word stem completion (Demb et al. 1995; Buckner et al. 2000).

How might the reduced conceptual/lexical priming evident under lorazepam be conceptualized? Once a geon structural description (GSD) of an object is activated by an object image in a naming task (a process not affected by lorazepam), the connection weights between units representing that particular GSD and units for the object's basic-level concept and name are strengthened (Cooper et al. 1992). The results of the present experiment suggest that lorazepam interferes with the strengthening of the later-stage connection weights.

In conclusion, neither lorazepam nor diazepam appears to exert deleterious effects on the early or intermediate stages in picture identification by which picture fragments might activate a structural description of an object's parts and relations. Instead, the interference produced by lorazepam on picture completion and concept- and picture-priming tasks can be isolated at a later stage where the association of a structural description of an object to its basic level concept of the object or its name is normally strengthened. This interpretation is consistent with the results from another study from our laboratory in which we used different pictures and a different way of measuring priming (Wagemans et al. 2000).

Acknowledgements This study was funded by the EEC Biomed2 grant (No. PL962775) to the first author and to a twin grant between France and Belgium to M. Boucart and J. Wagemans. The authors are grateful to Dr. Welsh, who performed the clinical examination of the participants, and to Tom Beckers and Eva Vandegaer who helped in the running of the tests.

\section{References}

Beckers T, Wagemans J, Boucart M, Giersch A (2001) Different effects of lorazepam and diazepam on perceptual integration. Vision Res 41:2297-2303

Bishop KI, Curran HV (1998) An investigation of the effects of benzodiazepine receptor ligands and of scopolamine on conceptual priming. Psychopharmacology 140:345-353

Biederman I (1987) Recognition-by-components: a theory of human image understanding. Psychol Rev 94:115-147

Biederman I, Cooper EE (1991) Priming contour-deleted images: evidence for intermediate representations in visual object recognition. Cognit Psychol 23:393-419

Brown MW, Brown J, Bowes JB (1989) Absence of priming coupled with substantially preserved recognition in lorazepaminduced amnesia. Q J Exp Psychol 41A:599-617

Bub ND, Black S, Hampson E, Kertesz A (1988) Semantic encoding of pictures and words: some neuropsychological observations. Cognit Neuropsychol 5:27-66

Buckner RL, Koutstaal W, Schacter DL, Rosen BR (2000) Functional MRI evidence for a role of frontal and inferior temporal cortex in amodal components of priming. Brain 123:620-640 
Buffet-Jerrott SE, Stewart SH (2002) Cognitive and sedative effects of benzodiaepine use. Curr Pharm Design 8:45-58

Buffet-Jerrott SE, Stewart SH, Teehan MD (1998) A further examination of the time-dependent effects of oxazepam and lorazepam on implicit and explicit memory. Psychopharmacology 138:344-353

Cooper EE, Biederman I, Hummel JE (1992) Metric invariance in object recognition: a review and further evidence. Can J Psychol 46:191-214

Curran HV, Gorenstein C (1993) Differential effects of lorazepam and oxazepam on priming. Int Clin Psychopharmacol 8:37-42

Danion JM, Peretti S, Grange D, Bilik M, Imbs JL, Singer L (1992) Effects of chlorpromazine and lorazepam on explicit memory, repetition priming and cognitive skill learning in healthy volunteers. Psychopharmacology 108:345-351

Demb JB, Desmond JE, Wagner AD, Vaidya CJ, Glover GH, Gabrieli JD (1995) Semantic encoding and retrieval in the left inferior prefrontal cortex: a functional MRI study of task difficulty and process specificity. J Neurosci 15:5870-5878

Dundee JW, McGowan WAW, Lilburn JK, McKay AC, Hegarty JE (1979) Comparison of the actions of diazepam and lorazepam. Br J Anaesth 51:439-446

Elliott MA, Becker C, Boucart M, Muller HJ (2000) Enhanced $\mathrm{GABA}_{\mathrm{A}}$ inhibition enhances synchrony coding in human perception. NeuroReport 11:3403-3407

Giersch A (1999) A new pharmacological tool to investigate integration processes. In: Boucart $\mathrm{M}$ (ed) The neuroscience of perceptual integration. Visual Cognition special issue, 3-4, 267-297

Giersch A, Boucart M, Danion JM, Vidailhet P, Legrand F (1995) Effects of lorazepam on perceptual integration of visual forms in healthy volunteers. Psychopharmacology 119:105-114

Giersch A, Boucart M, Speeg-Schatz C, Kauffman-Muller F, Danion JM (1996) Lorazepam impairs perceptual integration of visual forms: a central effect. Psychopharmacology 126:260270

Greenblatt DJ (1981) Clinical pharmacokinetics of oxazepam and lorazepam. Clin Pharmacokinet 6:89-105

Hummel JE, Biederman I (1992) Dynamic binding in a neural network for shape recognition. Psychol Rev 99:480-517
Humphreys GW, Rumiati RI (1998) Agnosia without prosopagnosia or alexia: evidence for stored visual memories specific to objects. Cognit Neuropsychol 15:243-277

Legrand F, Vidailhet P, Danion JM, Grangé D, Giersch A, Van der Linden M, Singer L (1995) Differential effects of diazepam and lorazepam on repetition priming on healthy volunteers. Psychopharmacology 118:475-479

Mandelli M, Tognini G, Garattini S (1978) Clinical pharmacokinetics of diazepam. Clin Pharmacokinet 3:72-91

Schifano F, Curran HV (1994) Pharmacological models of memory dysfunction? A comparison of the effects of scopolamine and lorazepam on word valence ratings, priming and recall. Psychopharmacology 115:430-434

Sellal F, Danion JM, Kauffman-Muller F, Grangé D, Imbs JL, van der Linden M, Singer L (1992) Differential effects of diazepam and lorazepam on repetition priming in healthy volunteers. Psychopharmacology 108:371-379

Stewart SH, Rioux GF, Conolly JF, Dunphy SC, Teehan MD (1996) Effects of oxazepam and lorazepam on implicit and explicit memory: evidence for possible influences of time course. Psychopharmacology 128:139-149

Thiel CM, Henson RNA, Morris JS, Friston KJ, Dolan RJ (2001) Pharmacological modulation of behavioral and neuronal correlates of repetition priming. J Neurosci 21:6846-6852

Tulving E, Schacter DL (1990) Priming and human memory systems. Science 247:301-306

Vidailhet P, Danion JM, Kauffman-Muller F, Grangé D, Giersch A, van der Linden M, Imbs JL (1994) Lorazepam and diazepam effects on memory acquisition in priming tasks. Psychopharmacology 115:397-406

Vidailhet P, Danion JM, Chemin C, Kazes M (1999) Lorazepam impairs both visual and auditory perceptual priming. Psychopharmacology 147:266-273

Wagemans J, Meire I, Boucart M (2000) Benzodiazepine effects on primed picture fragment completion (Poster). 23rd European Conference on Visual Perception, Groningen, The Netherlands. Abstract published in Perception (Suppl) 29:12b 
Copyright $\odot 2002$ EBSCO Publishing 